\title{
Metacognitive profiles in Schizophrenia and Bipolar Disorder: Comparisons with healthy controls and correlations with negative symptoms
} Raffaele Popolo ${ }^{\mathrm{a}, \mathrm{b}}$, Elizabeth Smith $^{\mathrm{c}}$, Paul H. Lysaker ${ }^{\mathrm{d}, \mathrm{e}^{*}}$, Krizia Lestingi $^{\mathrm{a}}$, Francesca Cavallo
Luisa Melchiorre

${ }^{\mathrm{a}}$ Center for Metacognitive Psychotherapy, Rome, Italy

${ }^{\mathrm{b}}$ Studi Cognitivi, Modena, Italy

${ }^{c}$ Department of Psychology, Indiana State University, Terre Haute, IN, USA

${ }^{\mathrm{d}}$ Richard L. Roudebush VA Medical Center, Indianapolis, IN, USA

${ }^{\mathrm{e}}$ Department of Psychiatry, Indiana University School of Medicine, Indianapolis, IN, USA

${ }^{\mathrm{f}}$ Center for Metacognitive Psychotherapy, Pescara, Italy

*Corresponding author: Richard L. Roudebush VA Medical Center, Day Hospital 116H, 1481 W. $10^{\text {th }}$ Street, Indianapolis, IN 46202, USA. plysaker@iupui.edu (PH Lysaker)

Abstract

While deficits in metacognition, or the ability to notice and reflect upon mental states has been observed in schizophrenia and linked with poorer concurrent and future function, it is unknown whether these deficits are unique to schizophrenia. Accordingly, this study assessed metacognition using the Metacognitive Assessment Scale-Abbreviated (MAS-A) and the Metacognitions Questionnaire- 30 (MCQ-30) among 26 adults with schizophrenia, 23 with bipolar disorder and 23 healthy controls. Symptom levels of the psychiatric groups were assessed with the Brief Psychiatric Rating Scale. ANCOVA controlling for age and education revealed

This is the author's manuscript of the article published in final edited form as:

Popolo, R., Smith, E., Lysaker, P. H., Lestingi, K., Cavallo, F., Melchiorre, L., ... Dimaggio, G. (2017). Metacognitive profiles in Schizophrenia and Bipolar Disorder: Comparisons with healthy controls and correlations with negative symptoms. Psychiatry Research. https://doi.org/10.1016/j.psychres.2017.07.022 
that the schizophrenia group had lower scores on the MAS-A total and its subscales compared to the bipolar group and healthy controls. The bipolar disorder group also had lower MAS-A scores than the healthy control group. No group differences were found for the MCQ-30. Examination of symptom correlates revealed MAS-A scores were most commonly related to negative symptoms in both clinical groups. The total score and need for control subscale of MCQ-30 was related to total symptomatology and positive symptoms in patients with bipolar disorder. Correlations between the two measures of metacognition revealed that higher MAS-A scores were significantly related to lower scores on the Need to Control Thoughts MCQ-30 subscale.

Keywords: Schizophrenia; Bipolar Disorder; Metacognition; Social Cognition; Negative symptoms 


\section{Introduction}

The ability to think about mental states has been referred to as metacognition for over 40 years (Flavel, 1978). As research on metacognition has expanded, the construct has come to encompass a spectrum of activities which range from noticing discrete thoughts, wishes and feelings, being aware of attentional biases and ultimately integrating these phenomena into a more complex sense of oneself and others which is needed to negotiate psychosocial challenges (Semerari et al., 2003; Lysaker et al., 2013). Recently, metacognitive deficits have been seen as playing an important role in outcome in schizophrenia. Metacognitive deficits have been observed in schizophrenia in all phases of the disorder (Lysaker et al., 2014; Vohs et al., 2014; Hasson-Ohayon et al., 2015; Masse and Lecomte, 2015; MacBeth et al., 2016;) and are linked to poorer outcomes. For example, deficits in the ability to form and use complex representations of self and others has been found to predict poorer levels of daily functioning (Snethen et al., 2014; Bo et al., 2015), anhedonia in the absence of depression (Buck et al., 2014), lesser levels of subjective recovery (Kukla et al., 2014), prospective assessments of psychosocial functioning (Lysaker et al., 2010), intrinsic motivation (Luther et al., 2016), and negative symptoms (McLeod et al., 2014; Lysaker et al., 2015a). From another perspective (Wells, 2000), dysfunctional metacognitive beliefs have also been reported in schizophrenia. These include negative appraisals about the benefit of becoming involved in cognitive activities and heightened anticipation of the uncontrollability, and danger of thoughts (Sellers et al., 2016) and have been associated with positive symptoms (Baker and Morrison, 1998; Morrison et al., 2011), especially hallucinations (Varese et al., 2011; Hill et al., 2012; Austin et al., 2015).

While these studies regarding metacognition have offered promising insights into understanding the unique challenges of schizophrenia, less is known about how unique these 
deficits are to schizophrenia as opposed to being a general feature of serious mental illness. For example, it is unclear whether persons with schizophrenia have similar versus dissimilar problems with metacognition compared to other common kinds of disabling psychotic conditions such as bipolar disorder. Several studies have found that patients with bipolar disorder have deficits in the ability to think about the mental states of others (Wolf et al., 2010; Martino et al., 2011; Benito et al., 2013; Temmerman et al., 2015; Santos et al., in press). Two recent metaanalyses have indeed found that compared to non-psychiatric controls, patients with bipolar disorder have deficits in the abilities to recognize both motive and affective states in others (Samamé et al., 2015) and that these deficits are not merely a function of mood state (Bora et al., 2016). Of note, one recent longitudinal study has suggested that patients with bipolar disorder recover their capacity for thinking about others as they move towards a euthymic mood state (Ioannidi et al., 2015).

While there may be metacognitive deficits in bipolar disorder, there is reason to hypothesize that these are less severe than in schizophrenia. Tas et al. (2014) have compared the metacognitive functions of patients with schizophrenia and bipolar disorder and found that patients with schizophrenia have more severe deficits in some forms of metacognition (i.e., selfreflectivity), but not others, and that the metacognitive deficits of both groups had a different pattern or correlation with neurocognitive function. The generalizability of this study was limited by the sample being composed of participants in a state of remission and the lack of a healthy control group needed to gauge the magnitude of deficits. A meta-analysis of studies looking at the related construct of social cognition found that overall patients with schizophrenia had more social cognitive deficits compared to patients with bipolar disorder, however there was significant overlap in terms of those deficits (Bora and Pantelis, 2016). Concerning 
metacognitive beliefs, studies have shown elevated levels of maladaptive metacognitive beliefs among patients with bipolar and unipolar depression which were associated with ruminations, and worry (Batmaz et al. 2014) as well as anxiety and depression (Sarisoy et al., 2014).

Understanding whether metacognitive deficits are present in bipolar disorder and differ in schizophrenia has several important implications. For one, if the metacognitive profiles of these disorders differ, then different treatment strategies may be called for; further, the confirmation of metacognitive deficits in bipolar disorder might point to the possibility of adapting clinical interventions which have shown promise for enhancing metacognitive capacity for patients with other conditions such as schizophrenia (Lysaker et al., 2015b) and personality disorders (Dimaggio et al., 2015; Vohs and Leonhardt, 2016; Buck et al., in press).

To explore this issue, the primary aim of this study was to test whether there were differences in the metacognitive capacity of three groups: patients with schizophrenia, patients with bipolar disorder and healthy controls. We included two measures, which tap different domains of metacognition: the Metacognition Assessment Scale Abbreviated (MAS-A; Lysaker et al., 2005) which assesses the capacity to notice mental states, form integrated senses of self and others, to see the world as viewable from multiple perspectives and then to use that information in the face of psychosocial challenges, and the Metacognition Questionnaire (MCQ; Wells and Cartwright-Hatton, 2004) which assesses metacognitive beliefs, or beliefs related to worry, cognitive confidence and self-consciousness, and need for control. We included both these measures because each, as noted above, has been separately linked to outcome in schizophrenia. Further, each may differently affect outcome in either condition. Whereas more synthetic forms of metacognition may limit persons' abilities to understand what they want and why they react as they do in dealing with psychosocial challenges, metacognitive beliefs or 
difficulties attending to attentional biases may result in heightened confusion, hyperarousal and worry, degrading function according to a different route. We predicted that patients with schizophrenia would exhibit the poorest levels of metacognition on both measures compared to patients with bipolar disorder and healthy controls and that the metacognitive function of patients with bipolar disorder would be more impaired relative to that of healthy controls. We reasoned this would be the case, given parallel findings that both psychosocial and social cognition deficits are greater in schizophrenia than in bipolar disorder and that social cognitive and psychosocial function of both groups is less than in persons with no psychiatric illness (Bora and Pantelis, 2016).

The secondary aims of our study were to examine the symptom correlates of both measures of metacognition. Recent theoretical and empirical work suggests that a lack of complex and integrated ideas about self and others may reduce the meaningfulness of daily experience resulting in reduced motivation and avolition (Buck et al., 2014). We predicted that lower levels of synthetic metacognitive function in the clinical groups would be related to negative symptoms. While negative symptoms in patients with bipolar disorder have not been nearly as widely studied as in schizophrenia, there is evidence that they represent a barrier to recovery for this group (Strauss et al., 2016). By contrast, we predicted that dysfunctional metacognitive beliefs in the clinical sample would be related to higher levels of positive symptoms, anxiety and depression (Wells and Cartwright-Hatton, 2004). We reasoned that rumination with themes of worry and threat might activate a vicious cycle in which negative cognitions and affects would reinforce one another, potentially sustaining delusional ideas and hallucinations. 
Finally, we had the exploratory aim of examining whether the two measures of metacognition were associated with one another. Theoretically, while each taps a different part of the spectrum of metacognition, they might be expected to influence one another. For example, lower levels of synthetic metacognitive capacity might leave persons less able to notice and correct a maladaptive focus on worry, threat, or control. Alternatively, preoccupation with worry, threat or control may limit awareness of changes in mental states making it difficult to understand oneself and others in a complex nuanced manner.

\section{Methods}

\subsection{Participants}

Participants were 72 adults diagnosed with bipolar disorder $(n=23)$, a schizophrenia spectrum disorder $(n=26)$, and a group of healthy control participants $(n=23)$. Both clinical samples were comprised of adults in a non-acute phase of illness and were recruited from two different outpatient psychiatric units and received medication and supportive counseling. The diagnosis was made according to the criteria of DSM-IV-TR (APA, 2000) by treating psychiatrists who had decades of experience in the treatment of psychotic patients. Further demographic information is included in Table 1. Exclusion criteria for the clinical samples were the presence of a disability or cognitive impairment, neurological disorders, drug addiction in the last month, hospitalizations or medication changes in the last month. The non-clinical sample were recruited through advertisements in local newspapers and underwent a short clinical consultation with a psychiatrist in order to exclude any major mental disorders, drug or alcohol addiction or neurological condition.

\subsection{Instruments}




\subsubsection{Indiana Psychiatric Illness Interview (IPII; Lysaker et al., 2002). The IPII is a}

semi-structured interview that asks participants to describe their understanding of their mental illness and psychological challenges. The protocol for participants with vs. without mental illness differed somewhat from each other. First, all participants are asked to tell the story of their lives. Participants diagnosed with a mental illness were then asked whether they believed they have a mental illness, and about problems related to this, as well as how they felt about having a mental illness. The next set of questions concern how things may have changed since having a mental illness, such as cognitions, emotions, personality characteristics and psychosocial function. Next, participants with mental illness were asked about how they controlled their mental illness and in what ways it controlled their lives. Participants with mental illness were then asked how their condition both affects and is affected by others. Finally, participants are asked what may be the same or different for them in the future. Participants without a mental illness, were asked the same series of questions, however, by contrast, in place of being asked about a mental illness they were asked about an emotionally or psychologically challenging situation in the last several years. This involved asking them about problems related to an emotionally or psychologically challenging situation, as well as how they feel about having experienced this situation. The next set of questions concerned how things may have changed since this situation, such as cognitions, emotions, personality characteristics and psychosocial function. Next, participants without mental illness are asked about how they controlled that situation and in what ways it controlled their lives. Participants without mental illness were then asked how that situation both affects and is affected by others. Finally, participants are asked what may be the same or different for them in the future. Interviews are audiotaped at the time of the interview and are later transcribed. Interviews typically last for 30 to 60 minutes and are conducted by trained research assistants. 


\subsubsection{Metacognition Assessment Scale-Abbreviated (MAS-A; Lysaker et al., 2005). The}

MAS-A is a rating scale used to assess metacognitive capacity as it pertains to the abilities to form complex and integrated representations of self and others. It was adapted on the basis of the original instrument, the Metacognitive Assessment Scale (Semerari et al., 2003) for use in studying metacognition in IPII transcripts and reconceptulized metacognitive function as existing along a spectrum (Lysaker et al., 2005) with lower levels involving lesser amount of integration and higher levels involving greater levels of integration. The MAS-A contains four scales: Self Reflectivity, which refers to the ability to think about oneself (scores range from 0 to 9); Awareness of the Mind of the Other, which refers to awareness of others' mental states, such as thoughts and emotions (scores ranging from 0 to 7); Decentration, which refers to the ability to see the world from multiple perspectives (scores ranging from 0 to 3); and Mastery, which refers to the ability to use knowledge of self and others to respond to psychological challenges (scores range from 0 to 9). For all scales of the MAS-A, higher scores indicate the capability for performing more complex metacognitive acts. Evidence of reliability and validity includes have been reported elsewhere (Lysaker and Dimaggio, 2014). The MAS-A differs from other forms of metacognitive assessment in that it conceptualizes reflections about the self and others as involving a series of increasingly complex acts which vary according to the amount and types of information which can be integrated, rather than thinking of self-reflectivity and awareness of others as primarily a series of modular functions.

\subsubsection{Metacognitions Questionnaire-30 (MCQ-30; Wells \& Cartwright-Hatton, 2004).}

The MCQ-30 is a shortened version of the Metacognitions Questionnaire and contains 30 items. It measures metacognitive beliefs that are implicated in a range of mental health conditions. The MCQ-30 is a self-report measure and yields five subscale scores and a total score, with subscales 
including: Positive Beliefs About Worry, Negative Beliefs about the Uncontrollability and Danger of Worry, Cognitive Confidence, Cognitive Self-Consciousness, and Need for Control (i.e., necessity of controlling one's own thoughts). Responses are given on a 4-point Likert scale, ranging from do not agree to agree very much, and then sums of the subscales are combined for the total score. For all scores, higher values reflect more problematic metacognitive beliefs.

\subsubsection{Brief Psychiatric Rating Scale (BPRS; Overall and Gorham, 1962; Conti et al.,} 1999). The BPRS rates participants on a number of symptom domains. Each domain is rated on a scale of 1 to 7 , with 7 denoting the highest level of severity. The 18 -item version of the measure was used to derive five scales: Anxiety/Depression; Withdrawal/Retardation; Thinking Disturbance; Activation and Hostility and Suspiciousness.

\subsection{Procedures}

All procedures were approved by the local ethics committee. Following informed consent and evaluation of inclusion and exclusion criteria, diagnoses were confirmed by senior clinicians, according to non-structured clinical interviews and after staff meetings. Participants completed the MCQ-30. The IPII was conducted by a trained interviewer with at least a doctorate in psychology. IPII interviews were later transcribed and then rated by trained raters who had received formal training of the MAS-A by one of the authors of the original MAS; they were blind to other test scores and did not conduct or transcribe the interview. The BPRS ratings were performed by trained raters with doctoral degrees in psychology. Ratings were performed blind to MAS-A scores.

\subsection{Data Analysis}

Analyses were performed in four steps. First, we planned to compare the demographic characteristics of the three groups as well as symptoms measured using the BPRS. Second, we 
planned to compare groups on the MAS-A and the MCQ-30, controlling for potentially relevant covariates. Third, we calculated Pearson correlations to explore whether metacognition was related to symptoms. While we made predictions about the Withdrawal/Retardation, Anxiety/Depression, and Thought Disorder subscales of the BPRS, we examined the correlations of the other subscales for exploratory purposes. Additionally, given the number of planned correlations, we used two-tailed tests of significance despite making unidirectional predictions. Finally, we planned to calculate Pearson correlations of the MAS-A with MCQ-30 scales to explore the relationship between the two metacognition scales for the entire sample.

\section{Results}

Mean scores for participant demographics and BPRS scores for the two clinical groups are reported in Table 1. As revealed in Table 1, ANOVA revealed that the schizophrenia spectrum group was significantly younger than the other groups while the control group had significantly more education than the other groups. The schizophrenia group also had higher levels of symptoms than the bipolar disorder group. In a follow-up analysis, differences in symptom levels persisted after we accounted for age differences between the groups.

Given differences in ages and education, the variables were included in the next analyses when we compared groups on the MAS-A and MCQ-30. As revealed in Table 2, the schizophrenia group had significantly lower ratings of metacognitive capacity on all subscales and the total score of the MAS-A compared to the bipolar disorder and control groups. These differences persisted between the schizophrenia group and the bipolar disorder group after adding the BPRS total as an additional covariate. No significant differences were observed between the groups in the MCQ-30 subscales or total. 
Next, we explored the relationship between metacognition and symptoms in the schizophrenia and bipolar disorder groups separately using Pearson correlations. Groups were not combined given differences between groups on the BPRS and MAS-A. As revealed in Table 3, higher MAS-A total scores were related to lower levels of the Withdrawal/Retardation BPRS scale for both clinical groups. For the schizophrenia group, Self-reflectivity and Awareness of the Mind of the Other were the subscales most closely related to negative symptoms while for the bipolar disorder group, Self-reflectivity and Mastery were the subscales most closely related to negative symptoms. A higher total score for the BPRS was related to higher levels of the MCQ-30 total score and the Need for cognitive control subscale for the bipolar disorder group. Greater thinking disturbance scores on the BPRS were significantly related to lower Selfreflectivity scores on the MAS-A and higher Need for cognitive control on the MCQ-30 in the schizophrenia group.

Finally, Pearson correlations were calculated to assess the relationship of the MAS-A and MCQ-30 scores. As revealed in Table 4, the pattern of correlations suggested that, in general, higher MAS-A scores were significantly related to lower scores on the Need to control thoughts MCQ-30 subscale. A range of other more modest correlations was found for other subscales of these instruments, but did not appear to coalesce into a broader pattern.

\section{Discussion}

In this study we compared two forms of metacognitive functioning of patients diagnosed with a schizophrenia spectrum disorder, bipolar disorder and a group of healthy controls and explored whether metacognitive function was related to negative and disorganization symptoms in the clinical groups. As predicted, patients diagnosed with schizophrenia appeared less able to form complex ideas about themselves and others, to see the world from a decentered stance and 
to use metacognitive knowledge to respond to psychosocial challenges relative to the other two groups. Patients diagnosed with bipolar disorder appeared less able to form complex ideas about themselves and others, to see the world from a decentered stance and to use metacognitive knowledge to respond to psychosocial challenges relative to the healthy control group.

Concerning concrete differences between the clinical groups, the schizophrenia group was only partially aware of their own nuanced emotions and struggled to perceive discrete mental events in others. They could not see how events were unrelated to them, but were able to frame a plausible psychological problem without, however, any sense of how to respond to it. The patients in the bipolar disorder group, by contrast, were aware of their own nuanced emotions and the subjectivity of their thoughts but struggled to see the world as offering other than what they wanted. They struggled to perceive nuanced affects in others, yet they could imagine a world in which they were not the center, but not necessarily that events can be viewed from legitimately different perspectives. They were able to perceive how they faced a plausible psychosocial problem and in response, could adjust their behavior, but less so, their thinking. These findings are consistent with the hypothesis that patients with bipolar disorder experience certain forms of metacognitive deficits, which are generally less severe than those found in schizophrenia (Tas et al., 2014; Bora and Pantelis, 2016).

Also, as predicted, lower levels of the overall ability to form and use an integrated sense of mental states was related to higher levels of negative symptoms in both groups. This is consistent with other work suggesting that negative symptoms may arise from deficits in synthetic metacognitive abilities (e.g., Buck et al., 2014). Previous work also suggests the presence of negative symptoms in patients with bipolar disorder even without presence of psychotic symptoms (Strauss et al., 2016). For example, it may be that impairments in the 
abilities to make sense of mental states makes it more difficult for persons with bipolar disorder and schizophrenia to engage with the world resulting in finding withdrawal to be the most viable option. In patients with schizophrenia, social withdrawal seems to be directly related to the difficulty to construct a clear representation of the other's mind, while in bipolar patients, social withdrawal may be more related to difficulties using metacognitive knowledge to respond to psychosocial challenges. There are alternative explanations, however, which cannot be ruled out including that the correlations found here reflect the influence of other phenomenon or that negative symptoms cause metacognitive deficits.

Overall, metacognitive beliefs were also related to positive symptoms in the bipolar group with Cognitive control being related to positive symptoms in both groups. Consistent with earlier research (Morrison et al., 2011), it may be that the tendency to view one's own thoughts as needing to be controlled is a risk factor for positive symptoms. It is also possible that for patients with both schizophrenia and bipolar disorder, positive symptoms may lead persons to distrust their thoughts and to shift their focus to controlling those thoughts. Again, alterative explanations cannot be ruled out including that other factors are responsible for the observed relationships.

There were unexpected findings. Metacognitive beliefs did not differ statistically between the three groups. This may suggest that in the more severe forms of mental illness disturbances in metacognitive beliefs are equivalent. This could be a reflection of our relatively modest sample size, however. While the MCQ total scores of the schizophrenia and bipolar group were virtually identical, they were a half standard deviation higher than the healthy controls. This may suggest that with a larger sample statistical differences between the clinical groups and controls might emerge. Metacognitive beliefs were not significantly linked with anxiety. As with all 
negative findings, however, speculation should be taken as tentative and fodder for future research.

Results of our exploratory analysis also pose some potential questions for future research. In particular, the link between higher levels of overall metacognitive capacity and a lesser need to control individuals' thoughts could suggest that persons are more likely to be fearful of thoughts as they emerge if they are less able to integrate mental activities in general into an integrated sense of the self and others. It is also possible that fearing that mental activities could derail functioning makes persons less likely to attend to and integrate information. Future research is needed to explore these questions further.

There are limitations. Our sample size was modest and limited to patients involved in treatment. Analysis thus lacked power and replications is needed in larger samples. We did not assess duration of illness and there was an imbalance in the proportions of men and women between groups. Additionally, multiple correlations were performed and the risk of spurious findings increased. While raters were not told of participant's status it might also have been apparent from the content of their IPII whether patient experienced mood elevations or disturbances in cognition. This study was lastly cross-sectional in nature and future longitudinal research is needed to explore the causal relationships among the variables.

With replication, there may be clinical implications. If patients with bipolar disorder experience metacognitive deficits, treatments which address metacognition in schizophrenia (e.g., Lysaker et al., 2015a; Lysaker and Klion, in press) could be tailored to assist this group. In particular, we anticipate that the tailoring of such treatments would take into account differences in metacognitive capacity between groups. For example, in addressing self-reflectivity for patients with Schizophrenia, group therapists might focus on understanding affect whereas for 
patients with bipolar disorder, therapists might address the disconnect between need and reality. Given links with symptoms, it may also be that treatments which enhance synthetic metacognition may affect negative symptoms, while by contrast, treatment that addresses metacognitive beliefs may affect positive symptoms. More research is needed to explore these possibilities. 


\section{Funding}

This research did not receive any specific grant from funding agencies in the public, commercial, or not-for-profit sectors.

\section{Acknowledgments}

None 


\section{References}

American Psychiatric Association, 2000. Diagnostic and Statistical Manual of Mental Disorders, fourth ed, text rev. American Psychiatric Publishing, Washington, DC.

Austin S., Mors O., Nordentoft M., Hjorthoj C., Secher R., Hesse M., Wells A., 2015.

Schizophrenia and metacognition: An investigation of course of illness and metacognitive beliefs within a first episode psychosis. Cogn. Ther. Res. 39: 61-9.

Baker C., Morrison A., 1998. Cognitive processes in auditory hallucinations: Attributional biases and metacognition. Psychol. Med. 28(5): 1199-1208.

Batmaz S., Ulusoy Kaymak S., Kocbiyik S., Turkcapar M., 2014. Metacognitions and emotional schemas: A new cognitive perspective for the distinction between unipolar and bipolar depression. Compr. Psychiatry. 55(7): 1546-55.

Benito A., Lahera G., Herrera S., Muncharaz R., Benito G., Fernández-Liria A., Montes J.M., 2013. Deficits in recognition, identification, and discrimination of facial emotions in patients with bipolar disorder. Rev. Bras. Psiquiatr. 35(4):435-8.

Bo S., Kongerslev M., Dimaggio G., Lysaker P.H., Abu-Akel, A., 2015. Metacognition and general functioning in patients with schizophrenia and a history of criminal behaviour. J. Psychiatr. Res. 225(3): 247-53.

Bora E., Bartholomeusz C., Pantelis C., 2016. Meta-analysis of Theory of Mind (ToM) impairment in bipolar disorder. Psychol. Med. 46(2): 253-64.

Bora E., Pantelis C., 2016. Social cognition in schizophrenia in comparison to bipolar disorder: A meta-analysis. Schizophr. Res. 175(1-3): 72-8.

Buck, K.D., Vertinski, M., Kukla, M. In press. Metacognitive Reflective and Insight Therapy: Application to a Long-Term Therapy Case of Borderline Personality Disorder. Am J 


\section{Psychotherapy}

Buck K.D., McLeod H.J., Gumley A., Dimaggio G., Buck B.E., Minor K.S., James A.V., Lysaker P.H., 2014. Anhedonia in prolonged schizophrenia spectrum patients with relatively lower vs. higher levels of depression disorders: Associations with deficits in social cognition and metacognition. Conscious. Cogn. 29c: 68-75.

Cassano G.B., Conti L., Levine J., 1999. Brief Psychiatric Rating Scale (BPRS), in: L. Conti (Ed.), Repertorio Delle Scale di Valutazione in Psichiatria. SEE Editrice, Florence, pp. 249-252.

Dimaggio G., Montano A., Popolo R., Salvatore G., 2015. Metacognitive Interpersonal Therapy for Personality Disorders: A Treatment Manual. Routledge, New York.

Flavell J.H., 1979. Metacognition and cognitive monitoring: A new area of cognitivedevelopmental inquiry. Am. Psychol. 34: 906-11.

Hasson-Ohayon I., Avidan M., Mashiach-Eizenberg M.; Kravetz S.; Rozencwaig S., Shalev H., Lysaker P.H., 2015. Metacognitive and social cognition approaches to understanding the impact of schizophrenia on social quality of life. Schizophr. Res. 161(2-3): 386-91.

Hill K., Varese F., Jackson M., Linden D.E., 2012. The relationship between metacognitive beliefs, auditory hallucinations, and hallucination-related distress in clinical and nonclinical voice-hearers. Br. J. Clin. Psychol. 51(4): 434-47.

Ioannidi N., Konstantakopolous G., Sakkas D., Oulis P. The relationship of Theory of Mind with symptoms and cognitive impairment in bipolar disorder: a prospective study. Psychiatriki 26: 1727. 
Kukla M., Lysaker P.H., Roe D., 2014. Strong subjective recovery as a protective factor against the effects positive symptoms on quality of life outcomes in schizophrenia. Compr. Psychiatry. 55(6): 1363-8.

Lysaker P.H., Carcione A., Dimaggio G., Johannesen J.K., Nicolò G., Procacci M., Semerari A., 1995. Metacognition amidst narratives of self and illness in schizophrenia: Associations with insight, neurocognition, symptom and function. Acta. Psychiatr. Scand. 112: 64-71.

Lysaker P.H., Clements C.A., Placak Hallberg C., Knipschure S.J., Wright D.E., 2002. Insight and personal narratives of illness in schizophrenia. Psychiatry. 65: 197-206.

Lysaker P.H., Dimaggio G., 2014. Metacognitive capacities for reflection in schizophrenia: Implications for developing treatments. Schizophr. Bull. 40(3), 487-491.

Lysaker P.H., Dimaggio G., Carcione A., Procacci M., Buck K.D., Davis L.W., Nicolo G., 2010. Metacognition and schizophrenia: The capacity for self- reflectivity as a predictor for prospective assessments of work performance over six months. Schizophr. Res. 122(1-3): 124-30.

Lysaker P., Gumley A., Luedtke B., Buck K., Ringer J., Olesek K., Kukla M., Leonhardt B.L., Popolo R., Dimaggio G., 2013. Social cognition and metacognition in schizophrenia: Evidence of their independence and linkage with outcomes. Acta Psychiatr. Scand. 127(3): 239-47.

Lysaker P.H., Klion R. In press. Recovery, Meaning-Making, and Severe Mental Illness: A Comprehensive Guide to Metacognitive Reflection and Insight Therapy. NY NY: Routledge.

Lysaker P.H., Kukla M., Belanger E., White D.A., Buck K.D., Luther L., Firmin R., Leonhardt B., 2015b. Individual psychotherapy and changes in self-experience in schizophrenia: A 
qualitative comparison of patients in metacognitively focused and supportive psychotherapy. Psychiatry. 78(4): 305-16.

Lysaker P.H., Kukla M., Dubreucq J., Gumley A., McLeod H., Buck K.D., Vohs J., Minor K.S., Luther L., Leonhardt B.L., Belanger E.A., Popolo R., Dimaggio G., 2015 a.

Metacognitive deficits predict future levels of negative symptoms in schizophrenia controlling for neurocognition, affect recognition, and self-expectation of goal attainment. Schizophr. Res. 168(1-2): 267-72.

Lysaker P.H., Vohs J., Hamm J.A., Kukla M., Minor K.S., de Jong S., Donkersgoed R., Pijnenborg M., Kent J.S., Matthews S.C., Ringer J.M., Leonhardt B.L., Francis M.M., Buck K.D., Dimaggio G., 2014. Deficits in metacognitive capacity distinguish patients with schizophrenia from those with prolonged medical adversity. J. Psychiatr. Res. 55: $126-32$.

Luther L., Firmin R.L., Minor K.S., Vohs J.L., Buck B.E., Buck K.D., Lysaker P.H., 2016. Metacognition deficits as a risk factor for prospective motivation deficits in schizophrenia spectrum disorders. Psychiatry Res. 245: 172-8.

MacBeth A., Gumley A., Schwannauer M., Carcione A., McLeod H., Dimaggio G., 2016. Metacognition in first episode psychosis: Item level analysis of associations with symptoms and engagement. Clinical. Psychol. Psychother. 23(4): 329-39.

Martino D.J., Strejilevich S.A., Fassi G., Marengo E., Igoa A., 2011. Theory of mind and facial emotion recognition in euthymic bipolar I and bipolar II disorders. Psychiatry Res. 189(3):379-84

Massé M., Lecomte T., 2015. Metacognitive profiles in individuals with a first episode of psychosis and their relation to social functioning and perceived social support. Schizophr. Res. 166(1-3): 60-4. 
McLeod H.J., Gumley A.I., MacBeth A., Schwannauer M., Lysaker P.H., 2014. Metacognitive functioning predicts positive and negative symptoms over 12 months in first episode psychosis. J. Psychiatr. Res. 54: 109-15.

Morrison A., Gumley A., Ashcroft K., Manousos I., White R., Gillan K., Wells, A., Kingdon D., 2011. Metacognition and persecutory delusions: Tests of a metacognitive model in a clinical population and comparisons with non- patients. Br. J. Clin. Psychol. 50(3): 22333.

Overall J.E., Gorham D.R., 1962. The Brief Psychiatric Rating Scale. Psychol. Rep. 10: 799-812.

Samamé C., Martino D.J., Strejilevich S.A., 2015. An individual task meta-analysis of social cognition in euthymic bipolar disorders. J. Affect. Disord. 173:146-53

Santos J.M., Pousa E., Soto E., Comes A., Roura P., Arrufat F.X., Obiols J.E., 2014. Theory of mind in euthymic bipolar patients and first-degree relatives. J. Nerv. Ment. Dis. In press

Sarisoy G., Pazvantoğlu O., Ozturan D.D., Ay N.D., Yilman T., Mor S., Korkmaz I.Z., Kaçar O.F., Gümüş K., 2014. Metacognitive beliefs in unipolar and bipolar depression: A comparative study. Nord. J. Psychiatry. 68(4): 275-81.

Sellers R., Varese F., Wells A., Morrison A.P., 2016. A meta-analysis of metacognitive beliefs as implicated in the self-regulatory executive function model in clinical psychosis. Schizophr. Res. [Preprint] Available from doi:10.1016/j.schres.2016.09.032. [Accessed 2 Jan 2017].

Semerari A., Carcione A., Dimaggio G., Falcone M., Nicolo G., Procaci M., Alleva G., 2003. How to evaluate metacognitive function in psychotherapy? The metacognition assessment scale and its applications. Clinical Psychol. Psychother. 10(4): 238-61. 
Snethen G., McCormick B.P., Lysaker P.H., 2014. Physical activity and psychiatric symptoms in adults with schizophrenia spectrum disorders. J. Nerv. Ment. Dis. 202(12): 845-52.

Strauss, G.P., Vertinski, M., Vogel, S.J., Ringdahl, E.N., Allen D.N., 2016. Negative symptoms in bipolar disorder and schizophrenia: A psychometric evaluation of the Brief Negative Symptom Scale across diagnostic categories. Schizophr. Res. 170(2-3): 285-9. doi: 10.1016/j.schres.2015.12.014

Tas C., Brown E.C., Aydemir O., Brüne M., Lysaker P.H., 2014. Metacognition in psychosis: Comparison of schizophrenia with bipolar Disorder. Psychiatry Res. 219(3): 464-9.

Temmerman A., Sabbe B., Morrens M., 2015. Social cognition in bipolar disorder. Tijdschr. Psychiatr. 57(6):405-14.

Varese F., Barkus E., Bentall R., 2011. Dissociative and metacognitive factors in hallucinationproneness when controlling for comorbid symptoms. Cogn. Neuropsychiatry 16(3): 193217.

Varese F., Bentall R., 2011. The metacognitive beliefs account of hallucinatory experiences: A literature review and meta-analysis. Clin. Psychol. Rev. 31(5): 850-64.

Vohs, J.L., Leonhardt, B.L. 2016. Metacognitive Reflection and Insight Therapy for borderline personality disorder: A case illustration of an individual in a long term institutional setting. Journal of Contemporary Psychotherapy 46(4): 255-264

Vohs J., Lysaker P.H., Francis M., Hamm J., Buck K., Olesek K., Outcalt J., Dimaggio G., Leonhardt B., Liffick E., Mehdiyoun N., Breier A., 2014. Metacognition, social cognition, and symptoms in patients with first episode and prolonged psychoses. Schizophr. Res. 153(1-3): 54-9.

Wells A., 2000. Emotional Disorders and Metacognition: Innovative Cognitive Therapy. John Wiley \& Sons, Chichester. 
Wells A., Cartwright-Hatton S., 2004. A short form of the metacognitions questionnaire: Properties of the MCQ-30. Behav. Res. Ther. 42: 385-96.

Wolf F., Brüne M., Assion H.J., 2010. Theory of mind and neurocognitive functioning in patients with bipolar disorder. Bipolar Disord. 12(6):657-66. 


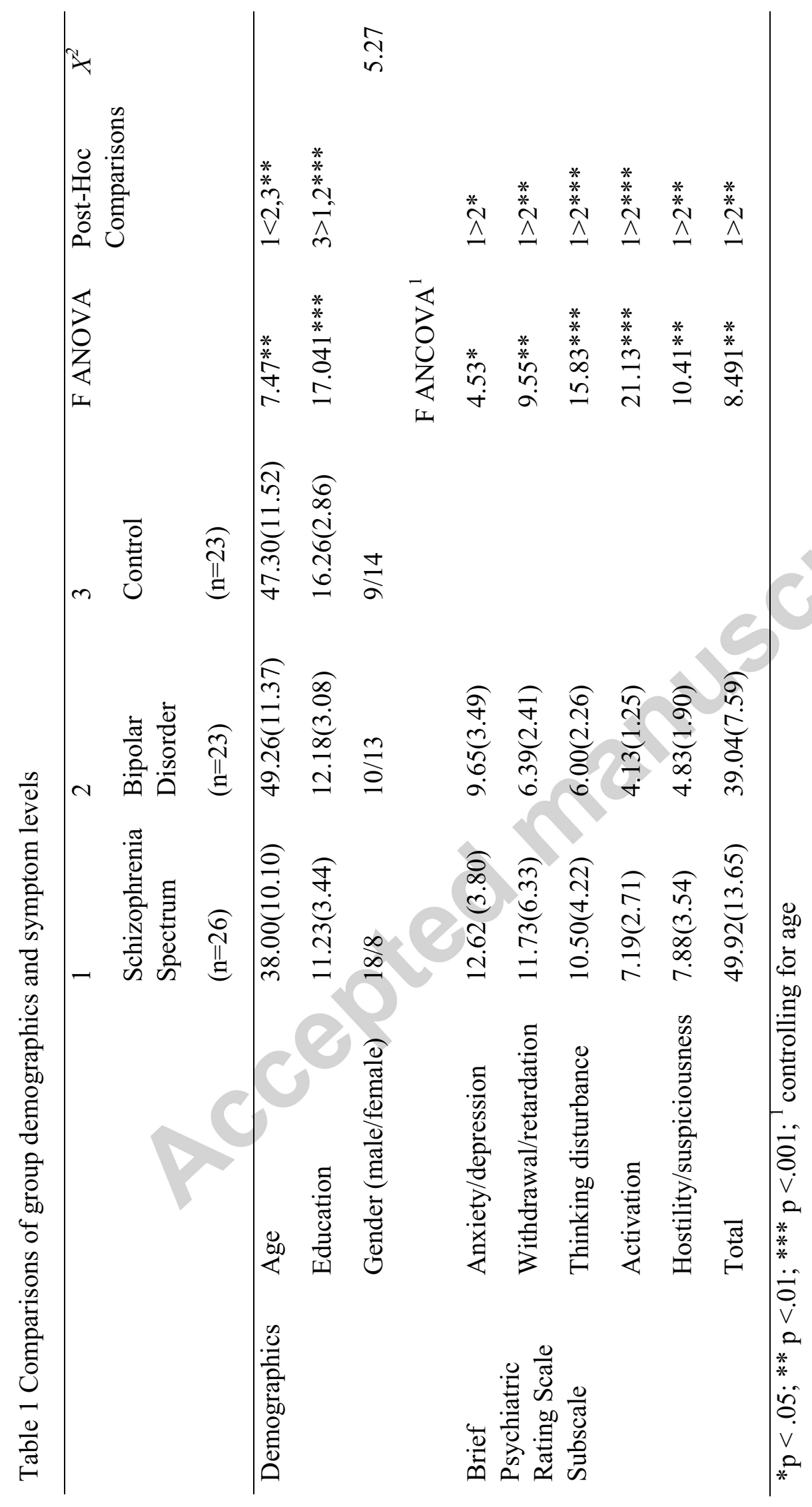


$\stackrel{\sim}{2}$

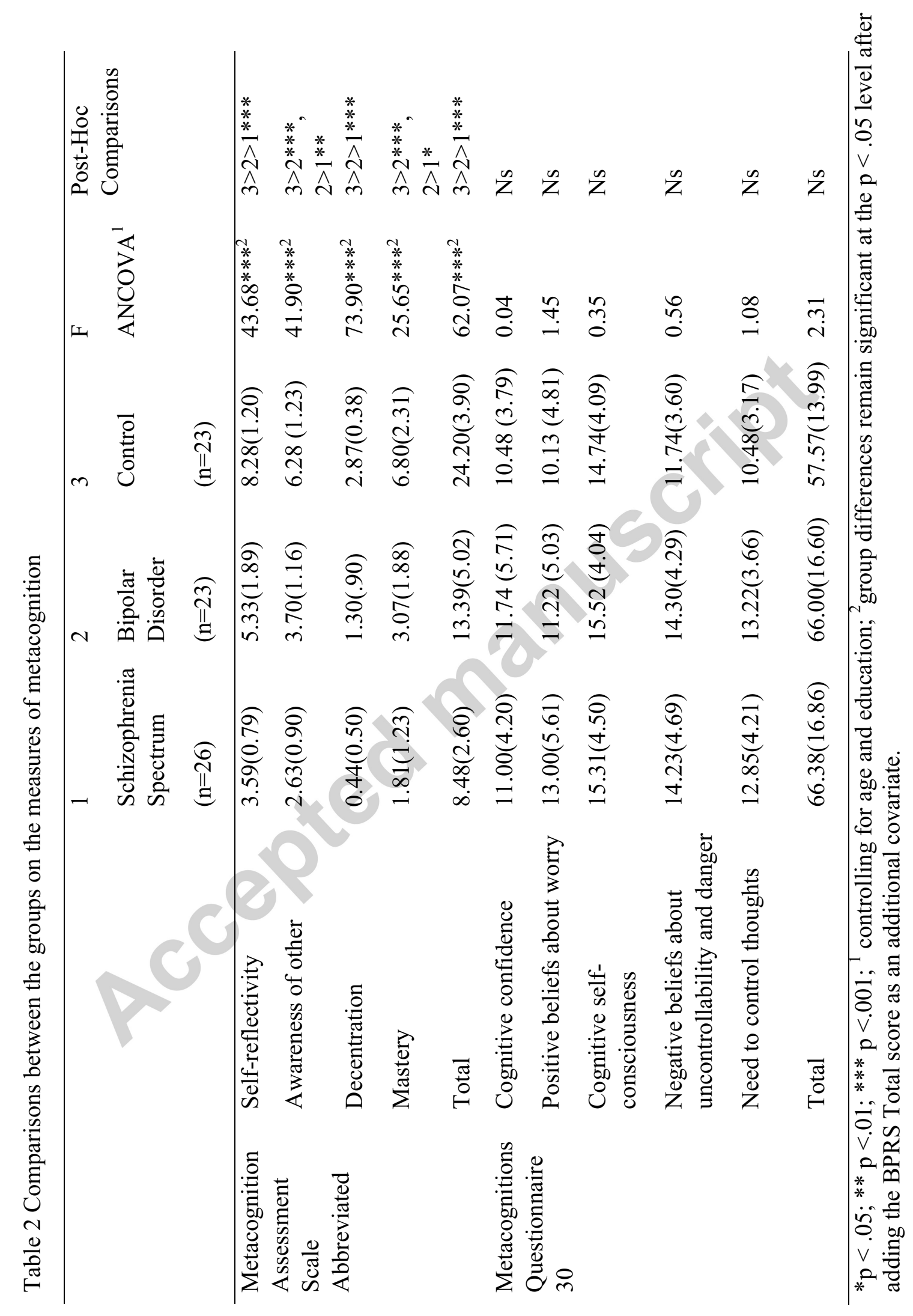




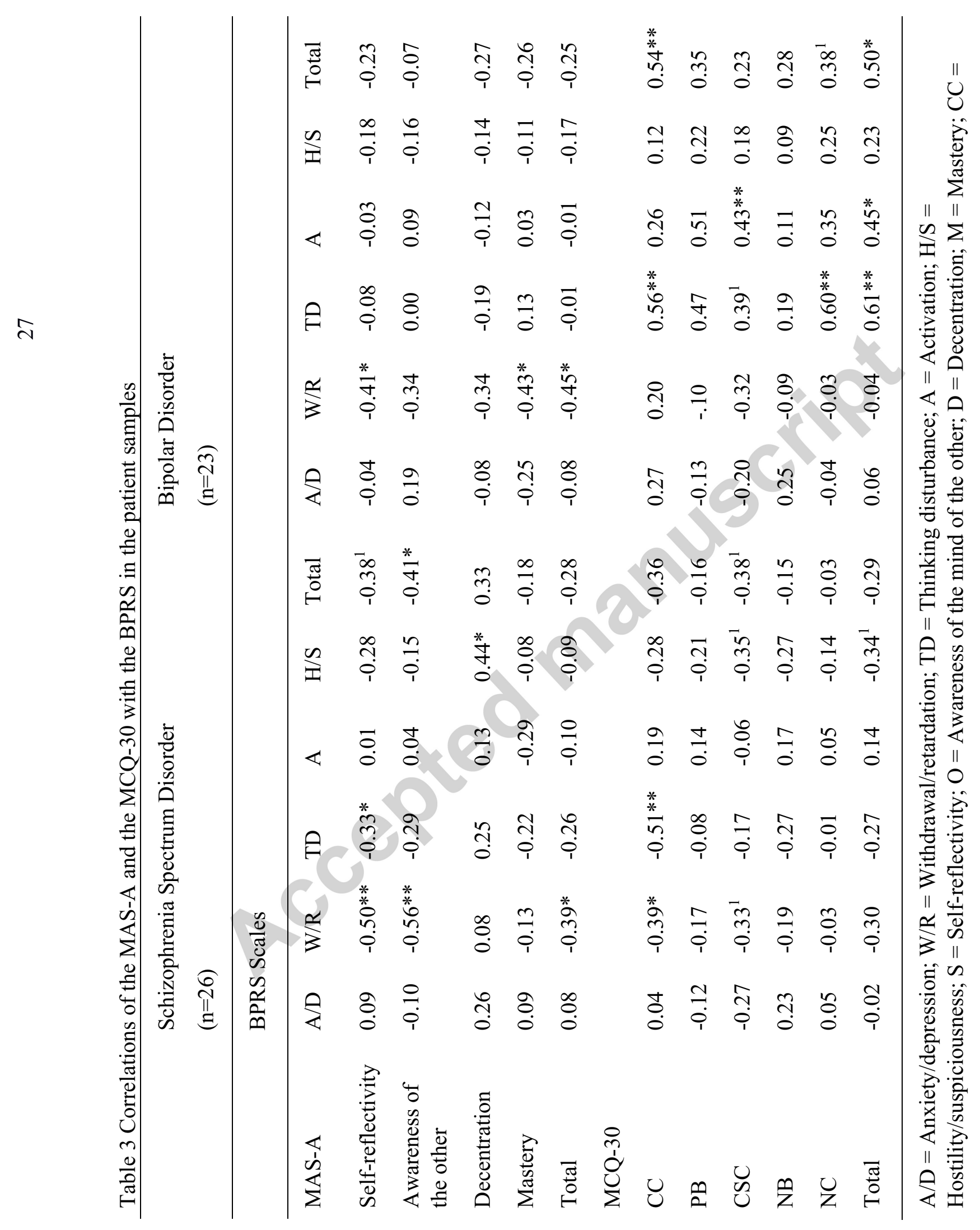


$\stackrel{\infty}{N}$

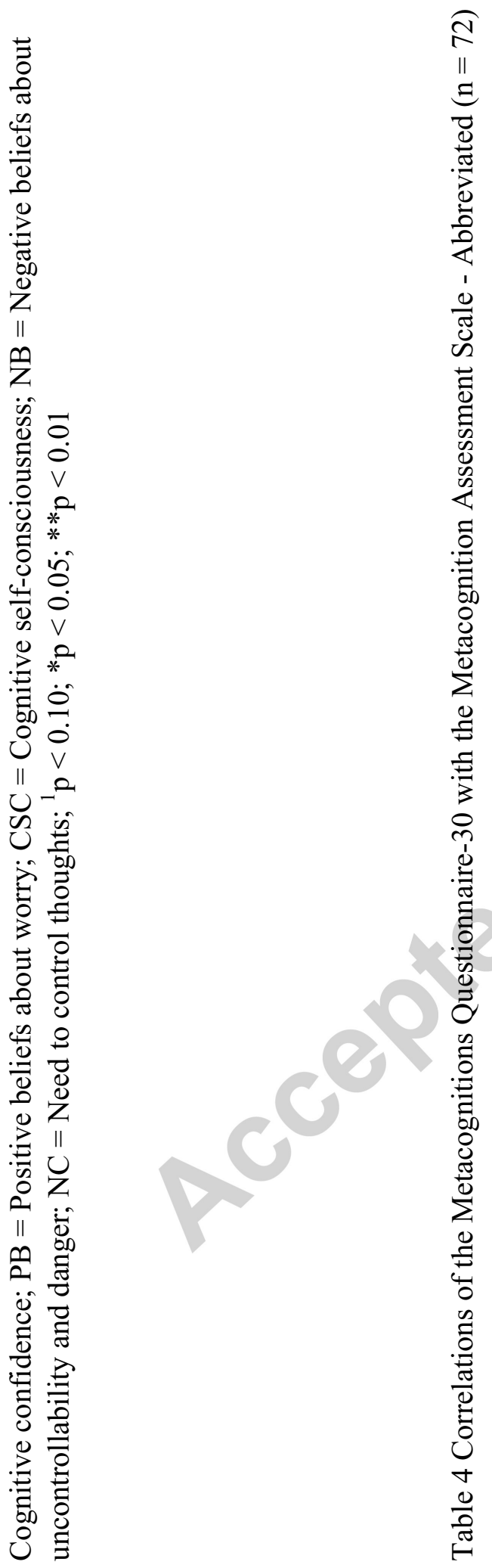




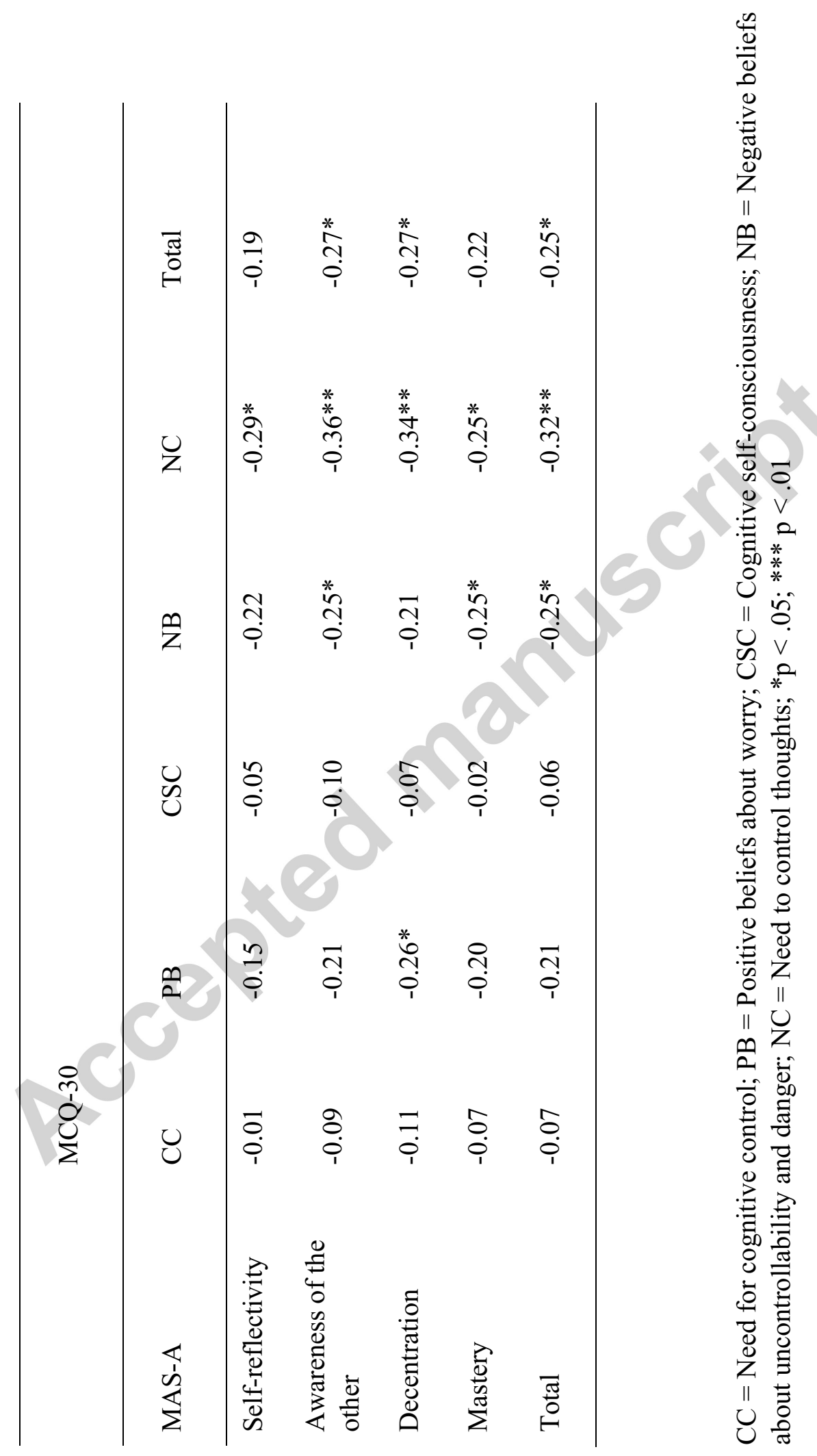


요

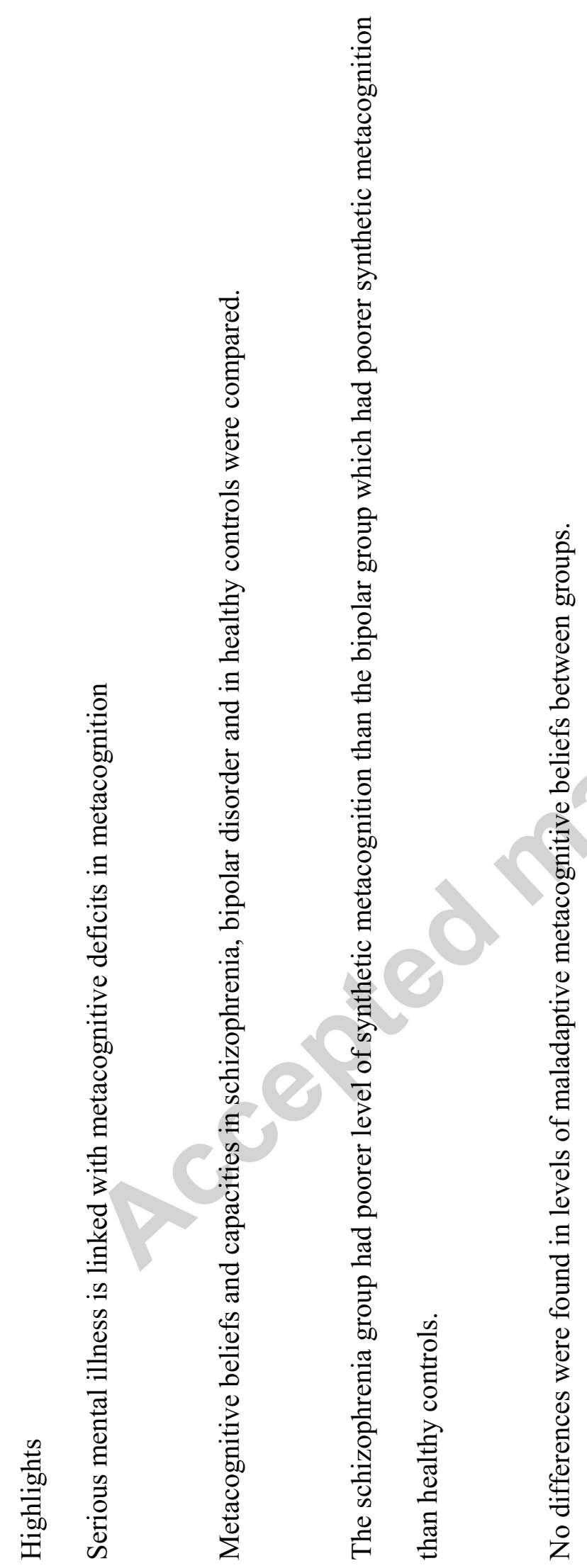

